\title{
Lapurdum
}

LAPURDUM Euskal ikerketen aldizkaria | Revue d'études basques |

Revista de estudios vascos | Basque studies review

$15 \mid 2011$

Numéro XV

\section{Pour une anthropologie du rire au Pays Basque}

\section{Eric Dicharry}

URL : http://journals.openedition.org/lapurdum/2315

DOI : 10.4000/lapurdum.2315

ISSN : 1965-0655

Éditeur

IKER

Édition imprimée

Date de publication : 1 octobre 2011

Pagination : 07-27

ISSN : 1273-3830

Référence électronique

Eric Dicharry, «Pour une anthropologie du rire au Pays Basque », Lapurdum [En ligne], 15 | 2011, mis en ligne le 15 octobre 2014, consulté le 19 avril 2019. URL : http://journals.openedition.org/

lapurdum/2315; DOI : 10.4000/lapurdum.2315

Dicharry E. | IKER 


\title{
Pour une anthropologie du rire au Pays Basque
}

\author{
Eric DICHARRY
}

Les occasions de rire pour les Basques ne manquent pas. Les moments propices se retrouvent dans les fêtes, dans le carnaval, dans les tobera, dans les chiquitos, dans les mascarades, dans les pastorales, dans la littérature orale, dans les contes, dans la mythologie, dans le théâtre comique, dans la littérature et nous avons choisi comme objet de cet article le bertsularisme et les mascarades souletines c'est en raison du fait que le contexte ludique que constitue l'improvisation orale au Pays Basque nord et le carnaval en Soule sont des cadres sociaux spatiotemporels privilégié de production et de circulation du risible et du rire des Basques. Un « temple » du rire et de l'humour où les Basques profitent de ces moments pour évacuer le sérieux de la vie quotidienne et pour parfois contester l'ordre établi.

Nous reviendrons en premier lieu dans cet article sur le choix et la légitimité de notre objet de recherche à savoir une anthropologie du rire des Basques au Pays Basque. Nous effectuerons ensuite un triple détour par une définition générale du rire, par le lexique de la langue basque qui consacre au rire une place importante et par une analyse des rapports entre d'un côté le rire et de l'autre la philosophie. Nous continuerons notre exposé par un voyage au pays des mascarades souletines et du bertsularisme en prenant des exemples concrets. Nous nous aiderons pour ce faire de textes tirés des phases qualificatives du championnat du Pays Basque nord des bertsularis qui s'est déroulée à Saint-Jean-Pied-de-Port en 2009. Nous conclurons cet article en évoquant les conditions nécessaires pour que le public composé des énonciataires / allocutaires des bertsularis puisse rire des énoncés chantés lors des joutes improvisées dans l'instant.

En guise d'introduction nous évoquerons le travail de Jean Duvignaud pour légitimer notre choix d'objet. Ce dernier, dans un ouvrage intitulé Le propre de l'homme, évoque la relation des anthropologues avec le rire et constate :

«Ethnologues, anthropologues ne parlent guère du rire. Sans doute se défient-ils du comique et des aspects hilarants de la vie commune? Il est vrai que la dérision trouble la cohérence des systèmes, la logique interne des structures ou la gravité des observateurs... Pourtant, les notes prises au jour le jour (quand elles sont publiées), les entretiens enregistrés, les photographies, les films montrent des moments d'hilarité qui s'effacent ensuite dans le discours élaboré ! (...) Létude des règles, des fonctions, des mentalités, des structures et de leurs combinaisons diverses, répond sans doute au ferme propos de définir la constance, la cohésion et la conservation des sociétés. 
Elle nous dit rarement comment les femmes et les hommes acceptent, subissent, contournent, déforment ces contrôles et ces prescriptions invisibles ou non qui définissent une culture. Nous ne savons pas grand-chose de la manière dont les vivants vivent la société... Dans le meilleur des cas, on nous renvoie au marginal, l'atypique, autant de termes avec lesquels, on tente de conjurer ce qu'on ne comprend pas. Et cela ne rend pas compte de la flânerie, de l'attente, du jeu, des passions, des moments inutiles de l'existence... Cela nous renvoie à une région inexplorée de l'expérience des hommes... Le comique, la dérision n'appartiennent-ils pas à cette région obscure et indéfrichée. » (Duvignaud, 1985, p 19)

Si le mot basque bertsularitza possède à l'intérieur de la culture basque un sens clair et précis : "Inprobisazio kontua esan nahi du. Herri poetak bat batean kantatzen du teknika eta arau tradizionalen arabera. Il s'agit d'improvisation. Le poète populaire improvise dans l'instant en fonction de techniques et de règles traditionnelles » (Euskararen liburu zuria, 172. or), il n'en est pas de même pour le rire et c'est sur sa définition que nous nous arrêterons maintenant.

Si nous suivons Eric Smadja dans sa définition qui utilise le concept positif et unificateur de communication, le rire est : "communication facio-vocale émettant des messages affectifs de plaisir, agressivité, angoisse (dénie ou non)». (Smadja, 1993, p 84). Pour le dictionnaire Robert, c'est « exprimer la gaieté par l'élargissement de l'ouverture de la bouche, accompagné d'expirations saccadées plus ou moins bruyantes ». Au niveau de ces manifestations physiques, il s'agit d'une expression faciale qui atteste la gaieté sous deux formes, l'une sonore, l'autre visuelle. Le rire se situe dans la troisième aire définie comme celle de Winnicott du schéma de la communication établie par Shanon et Waever. Cette dernière comporterait deux pôles, l'humour qui correspondrait au psychique-élaboratif et le comique au phénoménologiquereprésentatif. Eric Smadja note que :

« La communication s'établit entre un ou des émetteurs celui ou ceux qui font rire, produisant et transmettant le message risible à un ou des récepteurs, individus ou groupe, qui répondront par le rire, communication facio-vocale codée, agissant lui-même comme stimulus risible au sein d'une collectivité et exerçant un feed-back positif ou négatif sur le ou les émetteurs. Le message risible est véhiculé à travers différents canaux de transmission employant différentes modalités sensorielles (vision, audition, mais aussi tact voire rarement olfaction et goût). » (Smadja, 1993, p 113)

Les diverses expressions de la langue française comportant le verbe rire illustrent bien l'idée qu'il existe différents degrés. Du « rire à gorge déployée » au « rire intérieurement » en passant par « mourir de rire » et jusqu'à « rire aux larmes ». Polymorphe, le rire serait tout à tour « méchant, modeste, immodéré, bruyant » ou encore « de réprobation, du juste » ou tout simplement «fou ». Il pourrait de plus comporter une connotation positive (allégresse, gaieté) et inversement négative (moquerie, intérieur) comme en témoigne l'expression « rire de quelqu'un »...

Il renverrait de plus à d'autres catégories comme celle du sourire définie pas le Robert comme "prendre une expression rieuse ou ironique par un léger mouvement de la bouche et des yeux» ou encore celle de l'humour «forme d'esprit qui consiste à présenter la réalité de 
manière à en dégager les aspects plaisants et insolites ».

Mais laissons ici la langue française pour nous diriger dès maintenant vers l'univers sémantique de la langue basque et vérifier ensemble si le vocabulaire regorge lui aussi de mots qui caractérisent le rire. Nous tirerons nos exemples du dictionnaire Elhuyar et du dictionnaire du basque unifié Hiztegi batua publié par l'Académie de la langue basque Euskalzaindia.

Pour définir le rire, barre et irri sont utilisés par le dictionnaire Elhuyar. Pour le risible barregarri, irrigarri. La langue basque possède également le rire à gorge déployée irriz urratu et le rire aux éclats, algara egin, irribarrez ari, karkaraz egon. Pour plaisanter spécifiquement au Pays Basque nord : adarra jotzen ibili, bromotan, txantxetan, arrailerian ari izan. Pour la plaisanterie les basques utilisent : irri-barre, trufa, txantxa, adar-jotze, iseka, arraileria. Pour se moquer de : isekatu, trufatu, burlatu. Pour provoquer, attiser, taquiner, chatouiller ou faire la satire de : kitzikatu. Pour piquer, toucher et provoquer : zirikatu. Le basque connait de plus l'éclat de rire, algara et l'expression mourir de rire, algaraz lehertu.

La langue possède de plus la connotation négative évoquée précédemment avec la risée iseka, burla, trufa et pour se moquer, les Basques utilisent iseka, burla, barre, irri egin, trufatu. Pour désigner la satire et un mot piquant, c'est ziri qui est usité, pour faire une blague et se moquer, ziria sartu, pour désigner la vanne ou la pique piko, pour le sarcasme et la satire, eztenkada, et la « morsure des mots », hitzen klaska. Pour taquiner tentatu et pour définir un taquin, zirikatzaile, bihurri et un provocateur: kitzikatzaile.

Notons enfin pour terminer ce détour par la langue basque les références qu'en donne le dictionnaire du basque unifié Hiztegi batua publié par l'Académie de la langue basque Euskalzaindia chez la maison d'édition Elkar en 2008:

«Irri : irri-antzerki edo irri antzerki, irri-belar edo irri belar, irri egin, irri eragin, irrikarkaila, irribarre, irribarre egin, irribarrez, irribarreka, irribarretsu, irribera, irrikor, irrigarri, irrika, irrikan, irrikaz, irrikatu, irrikor Sin. irriberra, irrimarra Sin. Karikartura, irrintzi, irrintzi egin, irrintzika, irrintzilari, irrino Ipar. Sin. Irribarre, irris Sin. Arroz., irrisku, arrisku, irrist, irrist egin, irrista, irristada, irristagarri, irristailu, irristakor, irristalari, irristaldi, irristarazi, irristatu, irrits, irritsu, irriz, irrizko, irrizale».

Tous ces mots, verbes, proverbes et expressions étant autant de signes, de traces, d'indices du rire qui nous renvoie à une certitude première qui est que si les Basques ont élaboré un aussi riche vocabulaire spécifique pour qualifier le rire c'est qu'ils doivent le pratiquer.

La difficulté d'appréhender le rire découle de sa nature multiforme et hétéroclite, qui se révèle à première vue comme une réalité inclassable, dont il est malaisé de dégager l'unité puisqu'elle participe à la fois du psychique, du physiologique, du physique, de l'individuel et du social. Acte individuel et/ou collectif il est une réponse psycho-physique à un mécanisme psycho-physique (la Parole). Il est conditionné par la Langue qui résiste, en tant qu'institution sociale et contrat collectif, aux modifications de l'individu seul et il est en même temps porté par la Parole qui est combinaisons grâce auxquelles le sujet chantant peut utiliser le 
discours, c'est-à-dire le code de la langue en vue d'exprimer sa pensée personnelle. Aucun rire sémantique basque n'est possible s'il n'est prélevé dans le trésor de la langue basque. Son inexportabilité dérive de son intransidiolectalisabilité. Intimement lié par sa nature au domaine de l'idiolecte tel que Roland Barthes définit cette notion: «l'idiolecte comme le langage d'une communauté linguistique, c'est-à-dire d'un groupe de personnes interprétant de la même façon tous les énoncés linguistiques. »(Barthes, 1985, p. 26), le rire sémantique ne supporte guère le voyage transidiomatique. Lapproche qui soit la seule satisfaisante pour le saisir consiste à prendre en compte simultanément le faiseur de rire, le bertsu et le rieur. Car ce n'est pas dans le fabricant du rire, ni dans le texte isolé ni même dans le rieur que se trouve le lieu du phénomène du rire, mais dans une interaction entre le bertsulari, le bertsu et l'auditeur.

Nous n'irons pas jusqu'au point où veut nous entraîner Nietzsche quand il nous dit, à la fin de Par-delà le bien et le mal : "J'irais jusqu'à risquer un classement des philosophes suivant le rang de leur rire. » Nous n'avons pas comme Nietzsche de violente aversion pour ceux qui « ont cherché à donner mauvaise réputation au rire ». D’ailleurs, je ne me suis pas présenté aujourd'hui, devant vous pour dresser un quelconque classement. Ni de philosophes, ni de chercheurs en sciences sociales mais pour simplement vous communiquer les résultats de mes réflexions sur l'importance de porter une attention particulière sur le rire : de le prendre au sérieux. Attention spécifique non pas portée au rire en général mais au rire des Basques, au rire au des Basques au Pays Basque.

Mais avant de rentrer dans les détails de mes recherches penchons nous ensemble un instant, en guise d'introduction, sur l'attention portée sur le rire par les penseurs au cours de l'histoire. Pour ce faire nous reprendrons dans cette partie introductive une partie des données de la conférence prononcée à l'EHESS le 12 juin 2001 par Quentin Skinner intitulée La philosophie et le rire.

Nombreux ont été les penseurs qui au fil des siècles de sont intéressé au rire. L’un des premiers, et non des moindre, fut le philosophe grec du nom d'Aristote. Pour notre plus grand malheur, le Livre II de la Poétique, dont on sait qu'il portait sur la comédie fut perdu à la fin de l'Antiquité, et on ne sait rien de certain à son sujet. Ce qui est arrivé jusqu'à nous est la thèse d'Aristote qui se trouve dans le premier tome de la Poétique. Pour Aristote, le rire réprouve le vice en exprimant et en sollicitant des sentiments de mépris envers ceux qui se conduisent de façon ridicule.

C'est cette idée du rire comme expression de mépris qui a depuis été reprise par un grand nombre de penseurs. Cet intérêt pour le rire ne se dément pas et prend de l'ampleur au cours des premières décennies du XVlème siècle, en particulier chez des humanistes aussi éminents que Castiglione, Rabelais, Vives et Erasme. Lidée fondamentale de Castiglione - empruntée directement à Cicéron - est que les vices que nous pouvons espérer ridiculiser avec le plus grand succès sont ceux qui révèlent que nous avons ce qu'il appelle une vision « affectée » de notre propre valeur. Et il nous dit qu'il existe trois vices principaux de ce genre : l'avarice, l'hypocrisie et la vanité ou orgueil. (...)

A la fin du XVIe siècle, pour la première fois depuis l'Antiquité, nous voyons se développer 
une littérature médicale spécialisée concernant les aspects physiologiques ainsi que psychologiques de ce phénomène. Descartes consacre trois chapitres à la place occupée par le rire au sein des émotions dans son dernier ouvrage, Les Passions de l'âme, de 1648. Spinoza défend la valeur du rire dans le Livre IV de L'Ethique. Et nombre des disciples avoués de Descartes expriment un intérêt particulier pour ce phénomène, notamment Henry More dans son Account of Virtue. Mais bien avant cet intérêt pour le rire qui daterait du XVlème siècle, il faut remonter à l'Antiquité à Socrate, à Cicéron et à Aristote. La contribution des auteurs de la Renaissance à la théorie du risible est en réalité bien moins originale que ces auteurs ne voulaient l'admettre. Les humanistes avaient une dette considérable envers la littérature rhétorique des Anciens, et par-dessus tout, l'analyse de Cicéron dans De oratore.

Si ces penseurs se croient tenus de s'intéresser sérieusement au rire c'est que tous s'accordent sur une question cardinale : les émotions qui le provoquent. Une des émotions en question est une forme de joie ou de bonheur d'un genre bien particulier : la joie exprimée par le rire est toujours associée avec des sentiments de mépris, voire de haine. Chez les humanistes, l'un des plus anciens arguments à cet effet est avancé par Castiglione: "A chaque fois que nous rions, nous nous moquons de et nous méprisons toujours quelqu'un, nous cherchons toujours à railler et à nous moquer des vices. » De même, comme le souligne Descartes dans Les Passions de l'âme: "Or encore qu'il semble que le ris soit l'un des principaux signes de la joye, elle ne peut toutefois le causer que lorsqu'elle est seulement médiocre, et qu’il y a quelque admiration ou quelque haine meslée avec elle. » De même aussi Hobbes écrit, plusieurs années auparavant, dans The Elements of Law : "La passion du rire n'est rien d'autre qu'une gloire soudaine, et dans ce sentiment de gloire, il est toujours question de se glorifier par rapport à autrui, de sorte que lorsqu'on rit de vous, on se moque de vous, on triomphe de vous et on vous méprise. »

Au terme de ce détour par l'histoire de la pensée philosophique un constat s'impose : c'est le rire comme expression de mépris, le rire de, le rire contre, qui préside dans toutes ces réflexions sur le rire. Nulle trace ici ni d'un rire avec, ni d'un rire joyeux sans haine, ni d'un rire autocentré d'autodérision. Le rire positif est relégué au second rang loin derrière un rire à bannir, un rire qu'il faudrait au pire dissimuler, au mieux taire, un rire qui symboliserait une perte de contrôle de la maîtrise du corps : mal perçu. Comme le souligne Quentin Skinner :

"Notre histoire finit dans le cadre de ce que Norbert Elias a appelé le processus de civilisation, dont un aspect majeur fut, dans la culture européenne moderne, l'exigence croissante du contrôle par la volonté de diverses fonctions corporelles jusqu'alors considérées comme involontaires. Or le rire appartient de toute évidence à la classe des actions apparemment involontaires que les gens d'un tempérament raffiné se sont particulièrement souciés de contrôler. "

C'est pour confronter ces conceptions du rire à mon terrain d'enquête ethnologique que j'ai pris le parti de revisiter le rire en prenant appui pour ce faire sur le rire des Basques au Pays Basque à travers plusieurs recherches. La première s'est intéressée aux mascarades en Soule et a donné lieu à la rédaction d'un doctorat à l'EHESS. Travail récompensé par la Société d'Etudes Basques, Eusko Ikaskuntza par le prix de la Ville de Bayonne. La seconde, est en cours de rédaction. Elle est axée sur le bertsularisme contemporain. Pour cette étude, la Fondation José Miguel de Barandiaran m’a honorée de son financement. Je ne dévoilerai 
donc pas ici l'intégralité des résultats de mes recherches sur le sujet pour en garder la primeur à la Fondation, mais je prendrai ici tout de même appui sur quelques réflexions pour illustrer mon propos. La première thématique que je souhaite aborder avec vous concerne le rire dans les mascarades souletines.

\section{Le rire dans les mascarades souletines}

Je ne reviendrais pas dans le cadre de cet article sur l'intégralité des thématiques sur le rire élaborées lors de mon doctorat. Les auditeurs ici présents intéressés peuvent s’ils le souhaitent consulter ma thèse de doctorat dans les locaux de l'association Eusko Ikaskuntza de Bayonne. Mais je souhaite tout de même revenir un instant sur le rire des mascarades pour évoquer avec vous ce que je nomme, les limites du rire, en prenant un évènement qui s'est déroulé lors des mascarades de Barcus en 2009.

\section{Les limites du rire}

Je me suis rendu il y a peu dans le village de Barcus pour assister à la pastorale Xahakoa consacrée au chanteur Attuli et rédigée par Patrick Quéheille dit Kanpo. Dès mon arrivée dans le village, je conversais avec l'un des habitants bénévole qui avait été désigné pour placer les véhicules des visiteurs. Après avoir échangé brièvement sur mon origine j'évoquais avec lui mes études sur les mascarades. Ce dernier m'engageait sur le terrain des mascarades. Il me confiait que son fils avait joué le rôle de Pitxu dans les mascarades de Barcus de 2009. Pitxu me confiait-il c'est le clown des mascarades. Je ne savais pas véritablement ce qu'il voulait me dire mais j'allais vite être fixé. Lorsque les mascarades de Barcus se sont déplacées dans le village de haute soule de Larrau, continuait-il, mon fils s'est installé dans la voiture des gendarmes. Pour rire. C'est l'objet même des mascarades et du rôle de Pitxu que d'investir des espaces et des lieux. Effectivement, lors de mon séjour en Soule j'avais eu l'occasion de constater que Pitxu pouvait faire son apparition le jour de la représentation dans des lieux aussi divers qu'une salle de Mairie, une fenêtre d'une maison d'un habitant du village, le sommet d'un fronton... Tout l'art de Pitxu consistait précisément à dénicher des espaces et des lieux qui pouvaient procurer au public un sentiment de surprise. Plus la surprise était grande et plus il pouvait considéré qu'il avait bien fait son travail de Pitxu. Mais le jour de cette représentation de Barcus à Larrau, les choses n'allaient pas se passer comme d'habitude. Pitxu pris le parti d'investir le véhicule des gendarmes pour faire usage du klaxon. Avait-il dépassé les bornes du rire? Les gendarmes apparemment non au fait des procédures en cours lors de ce rite carnavalesque ne comprirent pas que cet usage du klaxon était destiné à faire rire, que c'était pour rire. Ils prirent ce tour pour une provocation. Enfin j'imagine. Il faudrait aller leur demander. Ils n'avaient semble t-il pas été sensible à l'art de Pitxu ! L'un d'eux décida alors d'agir. Son intervention consista à braquer Pitxu avec son arme en lui appliquant son arme à feu sur la tempe. Ce qui aurait pu passer pour une simple boutade, ce qui aurait du rester dans l'ordre du risible / comique / aurait pu se transformer en fait divers. Passage d'un registre à l'autre en raison d'une non compréhension du rite de la part des forces de l'ordre. Pour ces derniers, l'ordre devait primer même en temps de carnaval alors que ce temps est comme le reconnaissent tous les spécialistes en la matière celui de l'inversion et du désordre. En braquant Pitxu avec son arme à feu, le gendarme fixait au rite ses limites. Etaient marquées par cette fixation des limites du rite : les 
limites du rire. Le rire s'arrêtait sinon à la frontière du véhicule des gendarmes tout du moins à l'usage de leur klaxon. Le klaxon symbolisait un objet proscrit, interdit pour faire rire. La frontière du rire était dès lors matérialisée dans un avertisseur sonore.

Avec cet exemple se dessine les frontières du rire. Il illustre bien le fait que ce qui en fait rire certains ne correspond pas à ce qui en faire rire d'autres. La frontière délimite les deux espaces entre d'un côté ceux qui pouvaient rire de cet usage du klaxon des gendarmes, les jeunes barcusiens qui montaient la mascarade et une partie du public venu assisté aux mascarades et de l'autre les représentants des forces de l'ordre qui voyaient dans cet usage de leur klaxon et dans l'occupation de leur véhicule une défiance à ce qu'ils sont censés incarner en tout temps sans exception : l'ordre. Ainsi se dessine également qu'on ne peut pas rire avec tout, c'est-àdire ni avec tous les objets ni avec tous les espaces. Le véhicule des gendarmes échappait, en ce jour de visite des mascarades de Barcus à Larrau, au rite carnavalesque. Il imposait la limite à son rire en le censurant.

Mais il n'y a pas que lors des mascarades souletines que le rire est censuré. En dérangeant l'ordre établi, le rire dérange aussi le pouvoir, les institutions censées incarnées l'ordre, la sécurité, les valeurs. Souvenons-nous qu'il y a peu deux humoristes étaient licenciés de la radio France Inter pour avoir soit disons voulu poussé le rire à ses limites. Le licenciement d'un humoriste comme Stéphane Guillon est là pour rappeler au rirologue la causticité du rire. Que l'on aime où que l'on n'aime pas son humour, la question est précisément de cet ordre. Car le rire est révélateur d'identité, d'appartenances, de partage de valeurs. En effet, rire, c'est partager le temps du rire des valeurs, une manière d'envisager le réel. Et dans cette appréhension du réel lorsqu'il est question de rire, bien souvent deux camps s'opposent : ceux qui rient et ceux qui sont ris, ceux qui en rie et ceux qui n'en rie pas.

Après ce détour par les mascarades souletines partons maintenant pour le pays de l'improvisation. Ce second exemple puisé dans le bertsularisme viendra illustrer le rapport entre le rire et le sourire. Mais avant d'entrer dans le vif du sujet je voudrais insister sur l'importance du rire dans le bertsularisme. Le «faire rire » parait en effet être l'un des atouts majeurs des improvisateurs. Comme le note le célèbre Xalbador lorsqu'il évoque son compagnon Mattin.

"Je ne valais rien quand il s'agissait de faire rire. Même si, fatalement, j'ai essayé, cela ne m'a jamais vraiment réussi. En revanche, cela a toujours été l'atout majeur de Mattin. Moi-même, j'ai ri plus d'une fois à l'écoute de ses réparties. Heureusement, j'avais compris depuis longtemps qu'il valait mieux ne pas se vexer avec lui. Autrement, tant pis pour vous : il se réjouit de vous voir touché et vous en adresse de pires. Alors, vous n'avez d'autre solution que de commencer à rire, vous aussi. » (eke.org)

J'ai demandé à Xan Alkhat de me donner son point de vue sur ce thème du rire en lui posant la question suivante : "Orain dela bi aste edo hiru entzun nuen bertsualdi bat Gure Irratian, zahar bertsulariekin eta bertsualdia irriz beteta zegoen. Zuk uste orain lehen baino guttiago irri egiten dugu? ", "Il y a de cela une semaine ou deux, j'ai eu l'occasion d'écouter une session de bertsu sur la radio Gure Irratia avec d'anciens improvisateurs. Cette session était pleine de rire. A ton avis, riait-on plus autrefois qu'aujourd'hui ?». Sa réponse fut la suivante : 
« Bai dudarik gabe. Gero egoera edo bizitzak nahi duelako. Bainan ezin uka lehen desberdin zela. Jendea loriatzen zen bertsulariekin eta plazer haundia hartzen zuen heiekin irri egitea. Ene ustez, gutiagorekilan content agertzen zen lehengo publikoa, eta normal pixka bat, ideia xinpleenak, onenak baitira ardura. Bainan bertsoa garatu da, efektu gehiago sortu nahi dira orain. Bainan bada ere gaur egun, eta beharrik funtsean, publiko ona lekuka eta araberako bertsolari umorestuak ere. Bainan ezin gira beti umoretsu arizan bertsotan, gaiak hala nahi badu eta ideiak araberakoa lortzen badira orduan bai eginen da bertsoaldi umoretsua. Jendeak preziatzen du beti irri egitea eta irri eginarazten duten bertsolariak ere gustoko ditu. Iker Zubeldia ene ustez adibide ona da, ardura umore pitzarazle gaitza baita eta jendeak zinez maite du ene ustez." (Xan Alkhat, elkarrizketatuta)

"Oui sans aucun doute. Ensuite c'est aussi la situation et les existences qui en veulent ainsi. Mais on ne peut passer sous silence le fait qu'autrefois c'était différent. Le public et les gens se complaisaient, connectaient avec les improvisateurs et ils prenaient du plaisir à rire avec eux. A mon avis, le public d'autrefois était moins exigeant et il riait avec une production "normale ", avec des idées simples, celles-ci étant souvent les meilleures. Mais le bertsu s'est développé. Aujourd'hui des effets supplémentaires sont demandés. Mais aujourd'hui heureusement il existe toujours un public d'initié capable de réagir aux productions des improvisateurs ayant le sens de l'humour. Mais nous ne pouvons pas toujours faire de l'humour dans l'improvisation. C'est aussi fonction des sujets et des idées. Le public apprécie toujours le fait de rire et il apprécie également les improvisateurs qui les font rire. Iker Zubeldia est à ce titre un bon exemple du fait qu'il soit souvent capable de faire surgir l'humour et à mon avis, les gens aiment vraiment cela. (Entretien avec Xan Alkhat, novembre 2009)

\section{Rire et sourire dans une joute de bertsulari}

Pour Hobbes, il faut établir un contraste marqué entre le rire et le sourire. Pour le philosophe anglais, le rire exprime la dérision, mais le sourire est considéré comme une expression naturelle de plaisir, et en particulier d'affection et d'encouragement.

Nous allons maintenant évoquer une version toute différente de celle de Hobbes en démontrant grâce à un exemple puisé dans le répertoire bertsularistique que le contraste entre le rire et le sourire n'est pas autant marqué que Hobbes voulait bien le laisser entendre.

\section{Un curé à la mode...}

L'une des vidéos les plus consultées sur le site bertsotube.com est celle des qualifications pour la finale du championnat des bertsolaris du Pays Basque nord à Saint-Jean-Pied-de-Port ou s'affrontent dans une joute oratoire dans la salle du cinéma le Vauban Sustrai Colina et Maddalen Arzallus. Le sujet : le coiffeur et le curé. Un prêtre vient se faire couper les cheveux et demande une coupe moderne.

"Sustrai toi aujourd'hui tu es le coiffeur et Maddalen, un prêtre d'un certain âge. Elle vient te voir et te demande de le coiffer à la mode. » 
Nous constatons dès à présent sans pénétrer directement dans le bertsu que le thème choisi par le donneur de sujet peut permettre le comique, le rire à partir le l'antinomie entre la figure d'un curé d'un certain âge et l'univers de la mode qui est bien souvent éloigné des préoccupations des hommes d'église. Le couple (curé / mode) fonctionnerait tel un oxymore. Le sujet posé, c'est à l'Urrugnard Sustrai Colina de montrer ses capacités d'improvisateur.

Regardons ensemble si vous le voulez bien maintenant un extrait vidéo de cette joute.

Sustrai Colina commence son bertsu :

Utzirik Sakristia
tximista bezela
nigana etorri da
ezin zen bestela
nik kresta bat jarriko
nioke horrela
problema bakarra da
burusoila dela.

Pendant son bertsu, il a les deux mains dans les poches et au moment d'évoquer la crête, il sort sa main droite de sa poche et fait un signe, pomme ouverte, main dressée sur le crâne de manière à ce que le public perçoive de manière indubitable que la crête dont il parle ressemble à celle des jeunes au style quasi-punk. En évoquant cette crête et en la mimant il esquisse un léger sourire qui marque ses fossettes sur ses joues.

Comme le note Henri Bergson : "Jaloux de la parole, le geste court derrière la pensée et demande, lui aussi à servir d'interprète.» (Bergson, 1940, 2 ème éd, 2002, p24)

Mais ce léger sourire n'est qu'esquissé Il reprend très vite en un millième de seconde une posture beaucoup plus neutre et sérieuse pour terminer son bertsu comme il l'avait commencé. Comme s'il voulait adopter une distance vis-à-vis du contenu de l'énonciation. C'est que Sustrai Colina connaît l'importance de la maîtrise de soi. Il sait pertinemment que le simple sourire ne saurait lui être préjudiciable dans son énonciation.

Comme le note avec justesse Jean-Louis Maunoury : «(...) on sourit sans être dépossédé de soi » (Maunoury, 2001, p 14) Car le rire lui « saisit le rieur, le possède, ou plutôt le dépossède du contrôle de son corps et de son esprit, ce que des expressions telles que "fou rire " ou " mourir de rire dénotent bien. » (Maunoury, 2001, p 13) Il connaît l'importance de préserver ses idées claires en se préservant d'une empathie excessive. Comme le note Ernest Alkhat:

\section{«Ez da burua berotu behar sobera » (Alkhat, 2007, 54 orr).}

C'est d'ailleurs le reproche qu'il faisait au bertsulari Mendiburu qui avait lui tendance à trop se « chauffer les neurones». 
"Erraxtasun handia zuen, doinuetan ere. Baina burua sobera berotzen zuen. Txapelketek kalte egin diote". (Alkhat, 2007, 54 orr).

Comme le note Pello Esnal pour le bertsulari Manuel Olaizola Uztapide:

«Aurpegia beti izaten zuen serio, baina begiekin egiten zuen irri. Bertsotan beti serio agertzen zen, eta zaila zen igartzen noiz zetorren umore ukitua edo noiz ez. Aurpegia ez zuen apenas aldatzen eta. Horregatik igaro da diskretuago-edo".

C'est dans cet écart entre visage-corps et parole-discours que réside le secret de toute l'efficacité performative. Moins le visage est expressif et plus l'énoncé à de chance de toucher sa cible. Sigmund Freud note en ce qui concerne l'efficacité du mot d'esprit :

« Nous voyons cependant que l'auditeur témoigne de son plaisir par un rire qui explose, une fois que la première personne a prononcé le mot d'esprit, généralement avec une mine tendue par la gravité. »(Freud, 1988, p 266).

Plus le visage est sérieux plus le comique est efficace. C'est la distorsion entre ce qui est vu, un visage impassible tentant de maîtriser ses émotions dans un effort constant de retenue et ce qui est entendu, qui accentue l'effet comique. Le rire de l'improvisateur est tout au plus relégué dans le regard des yeux rieurs « begiekin irri » évoqués par Pello Esnal à l'endroit d'Uztapide, ou dans le léger et bref sourire de Sustrai Colina.

Nous avons réussi à expliquer l'un de nos questionnements, à savoir pourquoi le public ri alors que le bertsulari ne ri pas.

Ce dernier a pour sa part conscience du fait que l'efficacité de son rire est située dans la dernière partie du bertsu qu'il prononce à savoir dans burusoila qui se traduit par chauve, et il laisse à ce terme le soin de rejoindre la crête pour provoquer le rire du public. Crête (kresta) et chauve (burusoila) désormais réunies dans un même bertsu, initient l'effet comique recherché. C'est le contraste qui les sépare qui libère le rire. Le troisième vers pourrait donc se résumé par une flèche vers le haut, et le quatrième par une flèche vers le bas pour reprendre le parcours mnémonique confié par Mihura à Denis Laborde (Laborde, 1997, p. 42) Contraste qui constitue, avec l'image et le son, les trois entrées utilisées par le bertsulari Mihura pour retenir la fin du bertsu. La réussite du bertsu serait donc imputable au respect de ce contraste qui comme le note Mihura est la marque « des bons bertsu» qui «fonctionnent sur cette opposition positif/négatif, concret/abstrait, présence/absence. » (Mihura, in Laborde, op cit, p 42-43).

Dans l'exemple ci-dessus présence/absence de capilarité. Et l'effet ne se fait pas attendre, il est immédiat dès que le terme de burusoila est prononcé. Il s'accompagne de plus d'applaudissements fournis, preuve sonore de la réussite de son bertsu. L'effacement de son sourire est la pour renforcer la puissance du texte énoncé.

Limprovisateur à conscience de la direction de son propos puisqu'il connaît déjà la fin de 
son bertsu, il évolue « d'une indécision relative vers une conclusion préconstruite » (Laborde, 1997, p.39). Il commence toujours par la fin pour constuire son bertsu et tomber juste (cf, Denis Laborde, 1990). Comme le confie Mihura à Denis Laborde:

"Limportant dans tous les cas, c'est le dernier mot. Il faut toujours sortir quelque chose de surprenant là. Surtout si tu fais une joute contre un autre bertsulari, le dernier mot, c'est la pique, tu dois terrasser ton adversaire, le déstabiliser par rapport à ce qu'il aurait prévu de dire. L'important c'est donc ça : le dernier mot» (Mihura cité par Laborde, 1997, p 39)

Pour comprendre comment le rire se construit, il est primordial de saisir de quelle manière Sustrai Colina construit son bertsu. Si nous prenons en considération la méthode telle qu'elle figure dans le manuel du bertsolari de Xabier Amuriza intitulé Zu ere bertsolari, l'improvisateur passe par quatre étapes pour construire son bertsu.

«Gorago esana dugu lau urrats zeudela: 1) oinak. 2) Esaldiak. 3) Esaldiak erdibitu 4) Hornitu edo neurtu » (Amuriza, p 47)

1) Il définit les pieds: Bezela/ Bestela/ Horrela/ Dela

2) Il construit ses phrases: Sakristia tximista bezela/ Etorri da ezin zen bestela/ Kresta bat jarriko nioke horrela/ Problema bakarra burusoila dela

3) Il divise ses phrases en deux parties

Sakristia

tximista bezela

Etorri da

ezin zen bestela

Kresta bat jarriko

nioke horrela

Problema bakarra

burusoila dela

4) Il fournit et mesure

Utzirik sakristia

tximista bezela

nigana etorri da

ezin zen bestela

nik kresta bat jarriko

nioke horrela

problema bakarra da

burusoila dela. 
Sustrai Colina a déjà en tête, lorsqu'il débute son bertsu, l'image de son curé chauve, burusoila. Il ne connaît par contre pas, par avance, l'association burusoila/kresta puisque lorsque nous l'avons interrogé sur ce bertsu il nous confiait :

«Bertsoan beti pentsatzen da bukaera lehenik. Kasu hortan apezaren estereotipoa etorri zitzaidan, eta horrekin jokatu nuen. Gero azkena pentsatu, errimak atxeman, eta aitzina. "

"Pour improviser je pense toujours à la fin en premier. Dans ce cas précis, c'est le stéréotype du curé qui m'était venue et j'ai construit mon bertsu avec elle. Ensuite, j'ai pensé à la dernière, j'ai trouvé les rimes et je me suis lancé.»

Il n'a donc pas en mémoire le couple burusoila/kresta avant de se lancer. Suivons maintenant pas à pas le déroulement du bertsu. Au début, après avoir inventé le dernier vers et les rimes, il se concentre sur l'invention et l'énonciation des deux premiers vers. Mais dès qu'il arrive à la fin du troisième vers, il commence à sourire.

Ouvrons une parenthèse. Ce qui est intéressant, c'est que ce sourire est présent dans la vidéo que j'ai visionnée sur le site bertsotube mais absent de la vidéo projetée ici. Ceci est $\mathrm{du}$ au fait que ce n'est pas à un sourire entier que nous avons à faire mai à un demi sourire. Refermons la parenthèse.

C'est au moment même où il termine d'énoncer kresta qu'il sourit. S'il sourit c'est qu'il sait qu'il va coupler kresta avec burusoila, alors que le public l'ignore encore. C'est cette association qui le fait sourire (à moitié) et qui fera plus tard rire le public. Son cerveau a envoyé l'ordre aux muscles zygomatiques de se tendre. Son temps d'avance permet à son rire intérieur de devancer celui du public. Mais il ne veut pas encore tout dévoiler. Il mise tout sur l'effet maximal de la dernière partie de son bertsu. Son sourire s'efface aussi vite qu'il est apparu. Il se concentre sur son final. Rire à ce moment précis de l'énonciation aurait mis en péril son objectif premier : le rire de ses allocutaires. Pour arriver à ses fins, il met l'effet de son comique produit sur lui-même entre parenthèse, il étouffe et réprime son rire, pour mieux initier celui de son public. Son rire intérieur est un rire barricadé de l'intérieur.

L'objectif n'est pas pour lui de rire seul, mais de faire réagir le public en l'accompagnant sur le chemin du rire. Pour faire rire, le bertsulari s'interdit d'en rire. Tout au plus il s'autorise un léger sourire. Faire rire, c'est introduire par un effet signifiant, (les mots chantés entretiennent un rapport au symbolique), du désordre dans l'ordre des corps. Dès que le rire éclate, il suspend la parole, il l'a coupe à la source avec la suspension du souffle, c'est un fait physiologique. Comment rire, en effet, c'est-à-dire céder à ce petit spasme accompagné d'une expiration heurtée et continuer à proférer des mots ? C'est parce que le rire trouble l'énonciation en interdisant la parole chantée (celui qui rit ne peut à la fois rire et chanter), parce qu'il interfère dans la profération du bertsu que le bertsulari se l'interdit. Il est alors en face d'un choix. En rire, ou faire rire. Et c'est bien évidemment vers cette seconde solution qu'il penche. Le rieur va débarricader le rire que le bertsulari s'interdit. Le rire du rieur libère le rire $\mathrm{du}$ «faiseur de rire », il rend audible un rire inaudible, étouffé, barricadé, auto-interdit. Le bertsulari n'a plus besoin d'en rire car son rire est compris dans celui et/ou ceux qu'il(s) fait et/ 
l'avènement de l'insoutenable interruption pour l'autre »(Charney, 1997, p. 115)

Le rire n'est pas pré-existant à l'énonciation du bertsu mais, au contraire, il s'élabore pendant son énonciation. Il est concomitant à sa construction. Le «cousin du rhéteur romain (...) parent proche de Quintilien» (Laborde, 1997, p.42) a toujours un temps d'avance sur ses allocutaires et son rire intérieur devance de peu, mais devance tout de même, celui de son public. Il en ri, d'un rire retenu et inaudible, avant même de faire rire. Le rire de l'improvisateur basque est particulièrement paradoxal. Il est remarquable à la fois par sa présence et en même temps par son absence. La grave erreur serait alors de conclure, puisque leur rire est inaudible, que les bertsularis ne rient pas. Ce serait passé sous silence la signification de l'inaudible car ce qui ne peut s'entendre est loin d'être insignifiant. Ce n'est donc pas parce que le chercheur ne peut l'entendre qu'il n'existe pas (se référer ici au rire des rats inaudible à l'oreille humaine mais pourtant avéré, dans la note de l'introduction).

La stratégie comique vient s'imbriquer à celle mnémonique qui repose sur :

«(...) la gestion, dans l'instant de la profération orale, d'un ensemble d'éléments préexistants. Quels sont ces éléments? Les mots, la syntaxe d'une grammaire basque, une structure formelle (déterminée par le choix du support mélodique, du timbre), des sons, un schéma mnémonique qui lui permet de gérer cet ensemble d'éléments déterminés au moment de la profération ritualisée. » (Laborde, 1997, p.45)

L'élection de la structure formelle, consécutive à un commun accord des deux bertsulari qui participent à la joute oratoire improvisée, est elle-même partie prenante dans la réalisation de l'objectif. Choisir un air ou une mélodie non approprié créerait un décalage entre l'effet recherché (faire rire) et sa mise en branle.

Les commentateurs avisés ne manquent d'ailleurs pas de souligner ce décalage lorsqu'il est manifeste. Ce fut le cas par exemple lorsque les animateurs de la radio Gure Irratia critiquaient le choix mélodique d'une joute qui s'était déroulée en Soule en 2009 dans le cadre du prix Hernandorena.

Certains airs ou mélodies semblent donc plus propices au rire que d'autres, qui, elles, sont réservées pour faire vibrer le public sur des registres différents. Le bertsulari qui choisit d'émouvoir aux portes des larmes n'élira donc pas un air ou une mélodie traditionnellement réservée à faire rire. Le souvenir d'un air ou d'une mélodie peut rester associé au rire et influencer sa définition : un air qui a déjà fait rire sera prioritairement choisi pour refaire rire. A chaque émotion son registre mélodique ! Le choix de la mélodie par Sustrai Colina et Maddalen Arzallus n'est donc pas le fruit du hasard. Il est conditionné par le sujet qui leur est proposé. Un curé désirant se faire coiffer à la mode possédant en lui-même des prémisses de rire, les deux bertsulari adaptent leur choix au sujet. Ils font appel à leur mémoire mélodique. Ils se remémorent dans l'instant les dizaines de mélodies potentiellement opérationnelles et élisent, ensemble, parmi elles, celle qui sied le mieux à leur futur propos.

Le rire peut se focaliser sur la rime où s'exprimer dans une autre partie de l'énoncé. De 
plus il peut osciller entre rire phonique ou rire sémantique, ou compiler rire sémantique et phonique, le «nec plus ultra » du rire en quelques sorte. Ici, le couple Kresta/Burusoila ne se positionne pas en fin de vers. Rire et rime ne sont pas obligatoirement associés. Dans ce bertsu, certes les rimes occupent une place importante, défaillir à la règle compositionnelle aurait été considéré par le locuteur et ses allocutaires comme une grave faute de construction. Mais dans cet exemple, ce que le public retiendra en dernier lieu, ce sera d'en avoir ri. Lintérêt majeur de Sustrai Colina n'est pas centré sur les quatres rimes que sont Bezela/ Bestela/ Horrela/ Dela mais sur le couple antinomique Kresta/Burusoila initié par l'idée première, originelle, du stéréotype du curé. Ce bertsu peut donc, à juste titre, être considéré comme un contre exemple servant à revisiter les théories (concernant la représentation que se fait le bertsulari de son improvisation) proposées par Denis Laborde, en relativisant la suprématie du son et de la forme sur le sens des mots. Si l'observateur a parfois l'impression que l'aspect verbal de l'œuvre improvisée est moins organisée ou soignée (ou encore qu'elle subie la suprématie du son dans la représentation mentale que l'improvisateur se fait de son improvisation) que son aspect prosodique ou musical n'est-ce pas parce qu'il projette sur son objet « un point de vue de gens d'écriture » (Zumthor, 1983, p.127)?

Pour utiliser une image tirée de l'univers gastronomique, nous dirions que le son est tel un couvert qui permet d'apprécier la qualité des mets. Assiettes, couteaux, fourchettes, verres, serviettes, nappes, mobilier, décoration florale du lieu, mise en place de la table participent en grande partie à rendre l'expérience gustative inoubliable. Déguster les créations culinaires d'un chef étoilé dans des assiettes en carton serait du même ordre que de devoir se délecter d'un bertsu fameux mais bancal d'un point de vue mélodique, métrique ou rimique. Le grand bertsulari est pareil à un grand chef. Nombreux sont ceux qui proposent sur leurs tables du Pays Basque, des Merlu Koskera, mais bien peu sont ceux qui sont capables de le réinventer.

Limprovisateur basque est celui qui sait sublimer les ingrédients, celui qui, par son invention, sait associer tel mot à tel autre, celui qui est capable d'imprimer sa marque de fabrique, celui qui maîtrise l'art de la manipulation lexicale, syntaxique, grammaticale, mélodique et phonique, celui qui procure à la langue son style, avec sa saveur toute personnelle, inimitable, celui pour qui l'accord sémantico-formel est parfait, celui qui parvient à faire preuve d'esprit et d'innovation tout en respectant les règles, celui, enfin, qui est capable de conduire le public sur le chemin du rire.

Le choix de ses mots est autant sa signature que celui de ses rimes. Privilégier l'un sur l'autre serait une hérésie que le bertsulari n'a pas la faute de goût de commettre. Sens (fond) et son (forme) représentent les deux piliers de son architecture intérieure, l'une ne saurait subir de dommage sans porter préjudice à l'autre. Il ne saurait donc y avoir de suprématie du son sur le sens dans la représentation mentale que se fait le bertsulari de sa propre improvisation et si cette suprématie est pensée c'est qu'elle minimise la part d'inconscient contenu dans le dit hors rime. La rime donne le sens, la direction, la marche à suivre. Mais l'inverse est également vérifiable. Le sens peut lui aussi donner sa direction à la rime. Sens et son ne parviennent à l'aboutissement terminal (sémantique et sonore) de la performance et à l'effet recherché, qu'une fois imbriqués l'un dans l'autre. Si la suprématie du son sur le sens des mots est palpable et même énoncée par les improvisateurs eux-mêmes, elle ne peut qu'être 
momentanée, chronologiquement marquée dans la représentation que le bertsulari se fait de son improvisation. Instance passagère donc et non dernière instance. Le « faire rire » est doublement influencé par les jeux sonores et par la manipulation-formation du sens. C'est pour souligner cette double origine identitaire que nous utiliserons, pour préciser la place du sens ou du son dans le rire, les deux formulations suivantes: rire sémantique et rire phonique (se référer à la joie phonique de Paul Zumthor, 1983, p. 140) Le rire sémanticosonore sera attribué à un rire qui fait appel aux deux registres du sens et du son sans que nous puissions départager l'influence de l'un ou de l'autre. La préférence pour l'un ou l'autre de ces trois rires sera fonction du goût de l'énonciateur et de l'énonciataire. Certains préfèreront un rire sémantique (c'est la position de Sustrai Colina qui apprécie un rire nourri par le sens) d'autres par contre se contenterons d'un rire phonique. Ces jugements de valeurs du rire viendront s'ajouter à ceux que suscite l'improvisation et qui se fondent sur les qualités de la voix, la technique vocale de l'improvisateur ou sur le contenu du message. L'objectif n'est pas ici de contester que dans certaines circonstances, lorsqu'ils atteignent une "certaine intensité » (Zumthor, idem, p. 140) les échos ou les jeux sonores influent sur la formation du sens mais bien de souligner qu'ils ne constituent pas la dernière instance dans la représentation que le bertsulari se fait se son improvisation, en raison du fait que cette instance est variable.

Pour en terminer avec cette exégèse notons que Sustrai Colina n'utilise pas la deuxième personne pour qualifier le curé mais bien la troisième personne du singulier moins nominatif et plus général, il n'affronte donc pas directement son « adversaire », Maddalen en la laissant pour le moment de côté mais si c'est bien d'elle qu'il s'agit. Mais les termes d'adversaires ou de rival ne sont pas tout à fait propices à l'interprétation des relations inter-bertsolari.

Comme le laisse entendre Jon Enbeita dans l'un de ses bertsu donné lors de la finale du championnat de bertsolari à Saint Sébastien en 1982 :

« hemen ez dago kontrariorik

bat da besteen laguna"

(Bertsolari txapelketa, 1982, p 319) ici il n'y a pas d'adversaire (de contraire)

les uns sont les amis des autres

Entre les bertsolari il s'agit plutôt d'amitié fraternelle et comme dans toute relation amicale, il y a des taquineries, des rivalités internes mais qui restent bonne enfant. C'est de cela qu'il s'agit dans le cadre de la joute oratoire.

Voyons maintenant comment Maddalen Arzallus s'y prend pour construire sa réponse.

Nik krestari eman nahi
nioke senidan
inplanteen kontua
garatzen ari da
jarri nazazu azkar
modaren neurrira
ta agian serorak
hurbiduko dira.

Nik krestari eman nahi

inplanteen kontua

garatzen ari da

jarri nazazu azkar

ta agian serorak

hurbiduko dira.

\author{
Moi la crête je l'adopterai \\ tout de suite \\ c'est une question d'implants \\ et mets moi rapidement \\ à la mode \\ Et peut-être de la sacristine (religieuse) \\ ils se rapprocheront
}


Maddalen Arzallus reprend l'argumentation de Sustrai Colina concernant la crête. Elle invoque une terminologie contemporaine de la coiffure avec les implants. Elle confirme le pourquoi de sa visite, à savoir son désir de moderniser sa coupe de manière à suivre la tendance et enfin termine son bertsu en puisant dans le registre du religieux n'oubliant pas le rôle qu'elle tient, c'est-a-dire celui d'un homme d'église. C'est à la fin de son bertsu que Maddalen Arzallus intègre une dimension comique. Elle y accède en faisant référence à la sacristine. En imaginant un rapprochement entre d'une part le curé et d'autre part la sacristine elle renvoie le public à la question des relations que peuvent entretenir ces deux membres de l'institution religieuse. En proposant une liaison entre ces deux figures elle initie le rire, le public ayant bien à l'esprit le vœu de chasteté prononcé par ces deux personnages du monde religieux. Elle joue sur cette impossibilité en intégrant la dimension de relation possible, une relation interdite, sous entendant peut-être un rapprochement illicite basé sur des rapports sexués défendus. De plus elle ajoute à cette réunion une dimension transcendantale propre au discours religieux, en utilisant l'adverbe agian, qui signifie peut-être. Mais cet adverbe peut aussi se traduire par « plaise à dieu » et ce spécifiquement au Pays Basque nord comme le stipule la traduction tirée du dictionnaire Elhuyar. Agian: Plaise à Dieu. Agian jinen da! : plaise à Dieu qu'il/elle vienne!

Limprovisatrice joue dans ce cas présent sur un registre double ambivalent qui laisse au public la possibilité de choisir entre ces deux acceptions à savoir un simple peut-être où bien un plaise à Dieu. La question est bien ici de savoir s'il y aura une relation illicite entre d'une part le curé (apeza) et la religieuse (serora) et cet acte placé de plus sous la bénédiction de Dieu. Le discours blasphématoire ne se cache donc pas si loin, à peine dissimulé sous des insinuations. Mais Maddalen Arzallus se garde bien d'affirmer quoi que ce soit. Elle se contente d'évoquer, d'imaginer, de pointer du doigt. Libre ensuite au public de se faire sa propre interprétation des intentions du curé. Elle n'épuise pas le sens, elle initie des routes qui peuvent rester ouvertes aux analyses interprétatives. Elle puise dans le sujet qui lui est donné une interprétation toute personnelle, le curé voudrait se mettre à la mode pour mieux se rapprocher de la sacrisitine et ce grâce à sa nouvelle coupe, à ses implants, à son nouveau look pour employer une expression plus contemporaine puisée dans le vocabulaire de la langue anglaise et usitée dans le langage des jeunes générations. C'est à ce niveau que le rire puise ses racines.

Serorak lehendik ere
doaz Elizara
Beste proposamen bat
daukat hara hara
zurentzat bi inplante
ta Rasta gisara
Bob Marley berpiztea
errezagoa da

\author{
Les religieuses (sacristines) avant aussi \\ allaient à l'église \\ j'ai une autre proposition voila voila \\ pour toi deux implants \\ et à la mode Rasta \\ ressusciter Bob Marley \\ est plus facile
}

Sustrai Colina, rebondit sur la fin du bertsu de Maddalen en reprenant son terme de religieuse (serora) puis il fait une proposition de coupe au curé. Il lui propose deux dreadlocks, caractéristiques des membres de la communauté religieuse Rasta, qui est la contraction de Rastafari. C'est dans l'écart entre ces deux figures, d'un côté le curé et de l'autre le rastaman 
que l'improvisateur provoque l'humour du public. Imaginer un seul instant un curé avec des dreadlocks suffit à provoquer l'hilarité, d'autant plus que chez les rasta la pratique la plus communément admises et la consommation de chanvre indien plus connus sous son appellation latine de cannabis. Le public peut dès lors laisser fonctionner sans entrave son imagination et partir encore plus loin en se figurant un curé un pétard de cannabis en bouche. Le rire rend possible tout ce qui n'est même pas envisageable dans la vie courante, il crée un univers ou la permissivité est totale, ou l'imagination est reine.

E. Blondel note dans son ouvrage intitulé le risible et le dérisoire :

"Rire et jeu s'apparentent par le plaisir, la suspension du réel, la transgression des normes et la substitution de règles nouvelles, la liberté et l'enfance ou le retour de l'enfance. Le rire naît d'un hiatus, rupture du temps social sérieux et quotidien ». (Blondel, 1988).

Hors contraintes il se déploie et libère les individus de tous leurs présupposés, de leurs conditionnements. Limprovisation orale autorise le déguisement virtuel, la permissivité en repoussant les limites du conforme et de l'acceptable tout en envisageant le monde à parti d'une réalité. La consommation de cannabis est en effet très répandue chez les jeunes au Pays Basque. Tout en étant illégale sur le territoire national français elle est régulièrement pratiquée. Au Pays Basque sud elle est même largement tolérée et il n'est pas rare de sentir les effluves cannabiques dans de nombreuses tavernes. Rien d'étonnant donc que de retrouver ce cannabis dans la figure du rastaman à l'origine du rire. Limprovisateur puise dans une réalité qu'il connaît, qu'il fréquente et c'est en ce sens que la littérature orale reflète des pratiques sociétales. Sustrai Colina ne va pas jusque là, il se contente de proposer un curé accoutré en rasta mais de la à ce que le public jeune puisse imaginer un curé fumant du cannabis il n'y a qu'une étape que certains jeunes peuvent franchir aisément. Le bertsulari peut de la sorte dire plus que ce qu'il ne dit réellement. Il faut rechercher l'intérêt des jeunes générations à l'endroit du bertsolarisme dans le fait que l'improvisation leur parle de ce qu'ils vivent, de ce qu'ils connaissent déjà.

\section{Maddalen Arzallus}

Beraz hartuko duzu
orrazteko beta
baina ezaidazula
zuk egini xeka (?)
Bob Marlayen jarrita
daukazu areta
ezin dut meza eman
poroak erre ta.

Par conséquent tu vas prendre
du temps pour te peigner
mais dis moi
ton intention
transformé en Bob Marley
par ton soin
je ne peux pas donner la messe
après avoir fumé des pétards.

Maddalen Arzallus ne se prive pas de franchir l'étape évoquée plus haut dans le texte. Elle se saisit de cette opportunité qui lui est offerte par Sustrai Colina et précise qu'elle ne pourra pas officier après une consommation cannabique. On constate avec ce bertsu que tout l'art de l'improvisateur consiste à être attentif au contenu de son « rival-partenaire » afin de rebondir 
sur ses dires et en écho construire sa propre énonciation. C'est à cette capacité à répondre du « tac au tac » que se reconnait un bon improvisateur selon les bertsularis. C'est le dialogisme qui prime et qui opère en référence non seulement au sujet initial, le coiffeur et le curé, mais également aux thèmes évoqués pendant la joute. De sorte que l'attention du public est sans cesse réactivée. Une concentration partagée par les différents acteurs (improvisateurs, membres du jury, public) est donc intrinsèquement nécessaire. D'où l'obligation de silence de la part du public qui se doit de se plier à cette règle afin de ne pas interférer dans le bon déroulement de la « cérémonie ». La seule réaction autorisée outre les applaudissements et manifestations diverses (cris, acclamations, sifflets de considération, rires) étant celle de la reprise du dernier vers en accompagnement de la voix de l'improvisateur.

Nolako adorea
gure apezgaiak
baditu abildade
kemen ta dohaiak
hiru porro erretaz
Ze meza lasaiak!
Ba bai dena tapatzen du
intsentsu usaiak (Bis)

\author{
Quel ardeur (énergie) \\ notre séminariste \\ il a de l'habilité \\ de la vigueur et des aptitudes \\ une fois trois pétards fumés \\ Quelle messe tranquille! \\ oui, oui les usages d'encens couvrent tout
}

Sustrai Colina commence son bertsu en se basant sur une constatation qu'il tire de l'analyse de la performance elle-même. Ce n'est plus ici un thème puisé à l'extérieur, dans la réalité sociale qui est prise pour sujet mais bien la réaction du public qui réagit de manière particulièrement bien à la thématique du curé. Il rebondit de la sorte sur le succès du précédent bertsu de Maddalen Arzallus, il se place en observateur de l'évènement. Il poursuit dans cette logique par une énumération de ces qualités, habileté, vigueur, aptitudes (abildade, kemen, dohaiaik) puis revient sur la thèmatique du bertsu précédent en réactivant le pétard pré-énoncé. Pour terminer son bertu, Sustrai Colina avait la possibilité de choisir entre usainak (les odeurs) et usaiak (les usages) et il préfère la seconde possibilité. Une hypothèse pourrait consister à dire qu'il a souhaité adapter son discours au public en l'occurrence un public du Pays Basque nord et plus spécifiquement de Basse-Navarre (nous sommes ici rappelons dans la ville de Saint-JeanPied-de-Port) qui a l'habitude d'utiliser cette formule. Dans ce bertsu, une nouvelle fois il fait appel à une connaissance sur le sujet toute personnelle mais qui doit également être partagée par de nombreux jeunes qui souhaitent pouvoir fumer du cannabis clandestinement sans attirer l'attention. Pour ce faire recours à l'encens pour masquer l'odeur marquée et prégnante si caractéristique du chanvre indien. Ce choix n'étant évidement pas anodin puisqu'il renvoie une nouvelle fois le public à une terminologie religieuse qui correspond au sujet initial.

Erabat harritu naiz
gaurkoan zurekin
intsentsua ona da
nahi baduzu jakin
ilea luze utzi
ta koleta egin
ta meza emango dut
gitarra batekin (Bis)

\author{
Je suis totalement étonné \\ avec toi aujourd'hui \\ l'encens est bon \\ si tu veux savoir \\ laisse le cheveu long \\ et fais une couette \\ et je vais faire la messe \\ avec une guitare
}


Pour terminer cet article j'écrirai que j'ai parfaitement conscience qu'en m'attachant au rire des Basques je me suis attaqué à une des thématiques les plus complexes pour le chercheur en sciences sociales. Comme l'écrivait Pierre Bourdieu : « (...) une des choses les plus difficiles, pour un ethnologue, est de savoir ce qui est important ou pas important, ce qui est sérieux ou pas sérieux, la juste pondération des choses. » (Bourdieu, 2003) Lintérêt pour l'ethnologue de s'intéresser au rire dans la production bertsularistique est cependant incontestable. En effet, il permet de dépasser le discours officiel, convenu de la société qui présente à l'ethnologue les valeurs officielles du groupe pour accéder à la fois à l'Histoire, au passé refoulé et aux petites histoires empreintes de véracité qui sont comme l'écrit Pierre Bourdieu : «l'essentiel ». (idem, 2003) Tout change lorsqu'on casse le discours officiel des informateurs qui veulent faire bonne figure et lorsque le chercheur se réfère à des cas concrets en faisant sentir qu'il connaît les petites histoires. Lintérêt pour le chercheur de focaliser son attention sur l'improvisation versifiée dans l'instant c'est qu'elle permet de lui conférer une proximité avec le parler des choses de la vie ordinaire, avec des noms propres, des choses précises, et non avec de grandes déclarations vagues.

Avec cette recherche sur le rire, j'ai proposé au lecteur par le truchement d'une approche ethnolinguistique qui est un instrument de connaissance de soi très important, une sorte de psychanalyse sociale permettant de ressaisir l'inconscient culturel basque : " des structures mentales, des représentations, qui sont le principe de phantasmes, de phobies, de peurs (...) toutes les traces de la colonisation, l'effet des humiliations... (Bourdieu, 2003, p. 15) Avec Pierre Bourdieu je partage l'idée que les hommes, et c'est je pense universel, manipulent la réalité sociale qui existe en grande partie dans le discours. Ce discours du bertsulari porte en lui : " une part de vertu politique, dans la mesure où, dans la pratique, pour des raisons concrètes évidentes (politiques, sociales, culturelles) » (idem, p.15) un intellectuel poète basque « est missionné, sollicité dans le sens d'une recréation idéale de sa propre société, en particulier en réaction à l'image dévalorisante que tentent d'en donner ceux qui la nient » (idem, p.15). Limprovisateur basque contribue, en disant le monde basque et en faisant rire ses publics, à le faire exister. Comme l'écrit Pierre Bourdieu : "Dire que le monde, c'est ma représentation et ma volonté, quand il s'agit du monde social, ce n'est pas complètement fou, parce qu'il y a une élasticité du monde social, du fait que le monde social existe en partie par la représentation des gens qui y vivent (...). Le fait que les gens croient qu'un groupe existe, luttent pour qu'il existe contribue à le faire exister. » (idem, p. 17)

Nous terminerons cet article en laissant la parole aux créateurs et en particulier à Unai Iturriaga qui en 2002 à Saint Sébastien lançait :

$\begin{array}{ll}\text { Bertso polit bat bota didazu } & \text { Tu m'as envoyé un beau bertsu } \\ \text { ez nuen espero txarrik; } & \text { je n'en espérais pas de mauvais; } \\ \text { hona etorri ta erantzutea } & \text { venu ici et de répondre } \\ \text { ez da izango alperrik. } & \text { n'aura pas été inutile. } \\ \text { Barre egiteko ez dago baina } & \text { Pour rire il n'y a cependant pas } \\ \text { eskubideen beharrik; } & \text { besoin de permission; } \\ \text { barre egiteko behar dena da } & \text { out ce qu'il faut pour rire } \\ \text { hortako nahia bakarrik. (bis) } & \text { c'est juste l'envie d'en rire. }\end{array}$

(Bapatean, 2002, 79 or) 
Mais avant de conclure définitivement avec le rire, nous voudrions rebondir sur ce dernier bertsu. Pour en rire, nous pensons que l'envie est certes une condition nécessaire mais non suffisante. En effet pour réussir à rire, il ne suffit pas d'avoir envie de rire mais également et surtout de pouvoir en rire. S'il est si difficile à un non basque de pouvoir en rire ou de comprendre des bertsus c'est que ces derniers demandent une triple connaissance:

1) linguistique : il faut parler la même langue pour comprendre de quoi il s'agit. Une langue parlée qui dépasse le strict registre de tous les dictionnaires réunis.

2) contextuelle : il faut être capable de replacer les énoncés dans leur contexte

3) articulatoire par identité partagée : il faut non seulement être capable de réarticuler le détail à son contexte mais au-delà il faut qu'au terme de cette réarticulation il y ait consensus. Il faut que l'énonciateur (le bertsulari) et ses allocutaires (les auditeurs) tombent d'accord par connivence. Et c'est cette « connivence-accord » qui fait la différence. Seuls ceux qui partagent la même culture, c'est-à-dire la même manière de penser, de sentir et d'agir sont les plus à même à tomber d'accord sur ces détails qui font sens.

Une culture est bien plus longue à acquérir qu'une langue. C'est ce qui explique toute la difficulté et la complexité de toute recherche réalisée par des non basques à l'endroit de l'improvisation versifiée dans l'instant. C'est aussi à ce niveau qu'il faut trouver l'explication de l'orientation de l'objet même de ces recherches. C'est ce qui explique que ceux qui s'y sont dans le passé essayés ont tenté le plus souvent de passer par la marge, c'est-à-dire par la forme préférentiellement que par le centre c'est-à-dire par les détails compris dans le contenu.

Comprendre des bertsus, c'est-à-dire pouvoir vibrer pleinement lors de leur énonciation et parfois pouvoir en rire, ce n'est donc pas simplement une affaire de linguistique ni même de culture. Certains Basques partagent une même culture basque mais ne rient pas des mêmes choses. Au-delà de la culture, le rire est affaire de valeurs, de croyances, d'idéologie voire d'utopies partagées. En définitive, si les gens ne rient pas des mêmes choses, ce n'est pas uniquement parce qu'ils ne partagent pas une même langue où même une même culture, mais c'est essentiellement parce qu'ils ne partagent pas les mêmes valeurs, c'est-à-dire une même vision / conception / utopie du monde.

\section{Bibliographie}

Amuriza Xabier, Zu ere bertsolari, egin biblioteka, 1982, La Navarra.

Barthes, Roland, Laventure sémiologique, Paris, Editions du Seuil, 1985.

Bergson, Henri, Le rire : essai sur la signification du comique, PUF, Quadrige, lere édition 1940, 1 lème édition 2002.

Blondel, E, Le risible et le dérisoire, Paris, PUF, 1988.

Bourdieu, Pierre, Mammeri, Mouloud, Yassine, Tassidit, Actes de la recherche en sciences sociales, $\mathrm{n}^{\circ}$ 150, Du bon usage de l'ethnologie, 2003.

Charnay, Thierry, "Parole enfantine" pp. 109-120, in De la voix au texte. LEthnologie contemporaine entre l'oral et l'écrit, Dir, Nicole Belmont et Jean-François Gossiaux, 119è Congrès des Sociétés Historiques et Scientifiques, Amiens, 26-30 octobre 1994, Section 
anthropologie et ethnologie française, Editions du C.T.H.S, Paris, 1997.

Duvignaud, Jean, Le propre de l'homme. Histoire du rire et de la dérision, Hachette, Paris, 1985.

Esnal, Pello, Azaroaren 24an eta 25ean, Euskaltzaindiaren Herri Literatura batzordeak VIII. Herri Literatura Jardunaldiak egin zituen Donostian, Koldo Mitxelena Kulturunean, 2005.

Laborde, Denis, "Des images en des lieux, la mémoire d'un improvisateur", pp. 35-47, in De la voix au texte. L'Ethnologie contemporaine entre l'oral et l'écrit, Dir, Nicole Belmont et Jean-François Gossiaux, 119è Congrès des Sociétés Historiques et Scientifiques, Amiens, 26-30 octobre 1994, Section anthropologie et ethnologie française, Editions du C.T.H.S, Paris, 1997.

Lacan, Jacques, Le séminaire, Livre V, Les formations de l'inconscient, Seuil, 1998.

Maunoury, Jean-Louis, Le rire du somnambule; humour et sagesse, Seuil, 2001.

Smadja, Eric, Le rire, PUF, Que sais-je ?, n 2766, 1993.

Zumthor, Paul, Introduction à la poésie orale, Seuil, 1983. 